\title{
Geräteunterstützte Therapien bei fortgeschrittenem idiopathischem Parkinson-Syndrom
}

\author{
Device assisted therapies in advanced Parkinson disease \\ patients
}

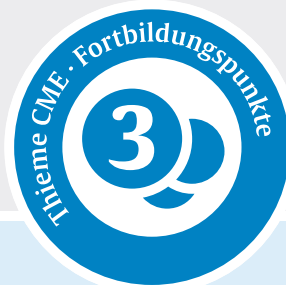

Andrés Ceballos-Baumann

Abteilung für Neurologie und klinische Neurophysiologie mit Parkinson-Fachklinik, Schön Klinik München Schwabing

\section{ZUSAMMENFASSUNG}

Wirkungsschwankungen der Dopaminersatztherapie gelten als eines der am meisten störende Symptome bei Patienten mit idiopathischem Parkinson-Syndrom (IPS) im fortgeschrittenen Stadium. Es besteht Konsens, dass bei Patienten, die Levodopa mehr als 5-mal täglich benötigen und trotz optimaler oraler levodopa- und nicht levodopabasierter oraler/transdermaler Therapien weiterhin schwere, störende Off-Phasen (länger als 1-2 Stunden am Tag) aufweisen, geräteunterstützte Therapien erwogen werden können. 3 Arten von geräteunterstützten Therapien (GUT) stehen zur Verfügung: die subkutane Infusion von Apomorphin, die Infusion in das Jejunum über eine perkutanen endoskopischen Gastrostomie (PEG) eines Levodopa/ Carbidopa intestinalen Gels (LCIG) mit oder ohne Entacapon (LECIG) und die tiefe Hirnstimulation (THS). Das Angebot an den äußerlich tragbaren batteriebetriebenen Medikamenten-Pumpen sowie der Systeme für die tiefe Hirnstimulation wird größer. Die Entscheidung, welche der GUT zum Zuge kommt, sollte in einem ausführlichen Abwägungsprozess zusammen mit dem Patienten und seinem Umfeld zustande kommen. Dabei sind die Präferenzen des Patienten und seiner Bezugspersonen sowie Alter, Verlauf des Parkinson-Syndroms, Ausmaß axialer Symptome, Komorbiditäten, neuropsychiatrischer und kognitiver Befund zu berücksichtigen. Die Entscheidungsfindung und die weitere Betreuung sollte idealerweise an Zentren stattfinden, die Erfahrungen in allen GUT haben.

\section{Einleitung}

Schwankungen in der Levodopawirkung während des Tages gelten als das am meisten störende Symptom bei Patienten mit 6 Jahren und längerer Krankheitsdauer [1] und sind die wesentliche Indikation für geräteunterstützte Therapien (GUT, englisch DATs für device assisted therapies). Zu diesen gehören äußerlich tragbare batteriebetriebene Pumpen mit Dopamin-Ersatzstoffen für subkutane und intestinale Infusionen sowie die tiefe Hirnstimulation (THS). Relevante Schwankungen in der Levodopawirkung treten nach den ersten Jahren des guten Ansprechens auf Levodopa nach 5 Jahren Levodopatherapie in ca. 20-40\% der Patienten auf [2]. Patienten bemerken eine kürzere, ungleichmäßigere und weniger zuverlässige Wirkung von Levodopa. Vor der nächsten Dosis von Levodopa kommt es zu einer Beschwerdezunahme („End of Dose Akinese“, „wearing off“). Beim „On-off“-Phänomen wechselt die Symptomatik innerhalb von Minuten bis plötzlich von einer guten Phase (On) zu einer schlechten (Off) Symptomkontrolle. Überdies kann es zu dosisabhängigen Dyskinesien während des Wirkmaximums von Levodopa in den On-Phasen (Peak-Dose-Dyskinesien) sowie beim An- und Abfluten von Levodopa im Blut kommen (biphasische Dyskinesien). Die Schwankungen in der Motorik sind nur der sichtbare Teil der Levodopaschwankungen, denn diese betreffen auch den Affekt, die Kognition, das Schmerzerleben und vegetative Funktionen.

\section{Indikation für GUT}

GUT, d. h. Medikamentenpumpen und THS, wurden entwickelt, um dem Problem der Wirkungsschwankungen der Dopaminersatztherapie zu begegnen. Bis vor wenigen Jahren galt, dass die Behandlungsmöglichkeiten mit oraler und transdermaler Medikation ausgeschöpft sein sollten, bevor eine Eskalation zu Pumpen oder THS in Betracht gezogen werden kann. Die Ergebnisse einer 2013 veröffentlichten großen Studie zur THS-Early-Stim-Studie [3] - hatte die Maxime der Ausschöpfung der konservativen Behandlungsmöglichkeiten für unter 60-jährige IPS-Patienten relativiert, sodass nach den Leitlinien von 2016 die THS des Nucleus subthalamicus (STN) Patienten schon in den ersten 3 Jahren nach Beginn von Fluktuationen oder Dyskinesien angeboten werden kann[4]. Leitlinien lassen für den Patienten und den behandelnden Neurologen einen breiten Ermessensspielraum, welcher Schweregrad der Symptome eine Indikation für eine GUT bedingt. In den letzten Jahren wird versucht, die Indikationsstellung sowohl für die THS als auch für die Medikamentenpumpen zu operationalisieren: Patienten, die Levodopa mehr als 5-mal täglich benötigen und trotz optimaler oraler levodopa- und nicht levodopabasierter Therapien schwere, störende Off-Phasen (länger als 1-2 Stunden am Tag) haben, sollten zur fachärztlichen Beurteilung mit der Frage für eine GUT überwiesen werden, auch wenn die Krankheitsdauer kürzer als 4 Jahre ist [5-7]. Aufbauend auf diese Empfehlung sind neuerdings die 5-2-1-Kriterien als Screening-Tool formuliert worden [8, 9]. Das heißt, wenn ein Patient mehr als 5 Einnahmezeitpunkte von Levodopa pro Tag braucht, 
um seine Symptome zu kontrollieren und mehr als 2 Stunden pro Tag im Off ist, also neben On-Phasen über 2 Stunden in einer Phase schlechter Symptomkontrolle (Off-Phase) ausharren oder 1 Stunde störende Dyskinesien erdulden muss, kann die Indikation für ein GUT näher erwogen werden. Das heißt nicht, dass eine Medikamentenpumpe oder die THS dann zum Einsatz kommen sollte, sondern nur, dass bei passender Indikation und Patientenwille diese erwogen werden können [4]. Die meisten Patienten wünschen außerdem, dass vor einer GUT alle konservativen Möglichkeiten versucht worden sind. Hinweise, was alles bei Patienten mit Wirkungsschwankungen versucht werden kann, ohne auf eine GUT zurückzugreifen, sind im Kasten „Therapieansätze“ aufgeführt.

\section{THERAPIEANSÄTZE}

Vor bzw. alternativ zu Medikamentenpumpen und THS bei Patienten mit Levodopawirkungsschwankungen

- Steigerung der Einnahmefrequenz von Levodopa

- Hyperfraktionierte Levodopa-Gabe

- als Levodopa/Carbidopa in Lösung zum Trinken

- Levodopa/Carbidopa 5/1,25 mg Microtabletten mit speziellen Dosierspender Schweden verbreitet

- Dietätische Maßnahmen: Proteinrestriktionsdiät (selten praktikabel), Proteinredistribution, kleine und häufige Mahlzeiten

- Kombinationstherapien von Levodopa mit MAO-B-Hemmer, COMT-Hemmer (inklusive Tolcapon) und Dopaminagonisten

- Bedarfsorientierte Gabe bei End-of-dose, wearing-off, delayed-morning-on, Off-Dystonien und unvorgesehenen Offs von in schnell wirksamer Darreichungsform als lösliche Tablette:

- Levodopa in sc

- Apomorphin-Pen

- Apomorphin-Film sublingual (in USA im Handel)

- Levodopa/Carbidopa zur Inhalation (in USA im Handel).

Potenziell ergibt sich eine Indikation für die aufwendigen, komplexen und kostspieligen GUT bei einer großen Patientenpopulation, wenn die Indikation für alle Patienten gilt, die mehr als 5 Einnahmen von Levodopa oral verteilt über den Tag brauchen. Gesundheitsökonomisch ergibt sich ein Szenario von kumulativen Kosten berechnet über 5 Jahre von ca. 90000 Euro für die THS, 140000 Euro für die Apomorphin-Pumpe und 230000 Euro für die LCIG-Pumpe, im Vergleich dazu das „best medical treatment" für 50 000-70 000 Euro [10]. Der Vergleich der Kosten der verschiedenen GUT untereinander ist aber schwierig, z. B. die THS-Patienten generieren bis auf die stereotaktische OP an sich, die THS-Hardware und die perioperative Versorgung wenige andere Kosten, denn sie sind in der Regel jünger und weisen eine kürzere Krankheitsdauer auf als die mit Pumpen versorgten Patienten. Nach einer Metaanalyse hinsichtlich Wirksamkeit und Kosteneffizienz unter besonderer Berücksichtigung möglicher Interessenkonflikten zeigten LCIG-Pumpe und THS eine höhere Wirksamkeit im Vergleich zu „best medical treatment“. Bei Betrachtung der Lebenszeitkosten war die Apomorphin-Pumpe günstiger als die THS und die THS günstiger als LCIG-Pumpe [11].

\section{Medikamentenpumpen}

Konzeptionell soll eine kontinuierliche dopaminerge Stimulation über gleichmäßige Plasmaspiegel des Pharmakons, die Einbrüche in der peripheren und zentralen Bioverfügbarkeit des Dopaminergikums verhindern und damit die vielfältige klinische Symptomatologie der Off-Phasen (z. B. motorisch, sensorisch, affektiv) lindern. Dies wird erreicht, indem über eine äußerlich tragbare batteriebetriebene Pumpe entweder subkutan der Dopaminagonist Apomorphin oder jejunal Levodopa/Carbidopa intestinales Gel bzw. mit Entacapon kontinuierlich infundiert wird ( $\triangleright$ Abb. 1, $\triangleright$ Abb. 2). Die Pumpensysteme unterscheiden sich je nach verwendetem Wirkstoff in Hard- und Software, Größe und Verabreichungsort. In Abhängigkeit von dem Pumpenmodell lassen sich Dosierungen und die Extradosen (Bolus) programmieren: Morgenbolus bei LCIG und LECIG, kontinuierliche Flussraten, bei manchen Pumpen unterschiedlich Flussraten gebunden an bestimmte Tageszeiten, Extrabolus zur Unterbrechung einer Off-Phase unter kontinuierlicher Flussrate sowie unterschiedlich lange Sperrzeiten für die Extradosen.

\section{Apomorphin-Pumpen zur subkutanen Infusion}

Bei der Apomorphin-Pumpe ist keinerlei Operation notwendig. Ähnlich wie bei Diabetikern mit einer Insulin-Pumpe muss einmal am Tag eine feine Nadel subkutan gestochen werden, die die Pumpe über einen Katheter mit der äußerlich getragenen Pumpe verbindet. Einige Patienten kommen auf die Pumpe, weil sie den Apomorphin-Pen für den bedarfsorientierten Einsatz schon kennen, etwa bei Offs morgens, bei Levodopa-„Blindgängern“, bei antizipierten Offs, bei rigorbedingten Schmerzen bis hin zu anhaltenden Muskelkontraktionen (Krämpfe) als Off-Dystonien sowie bei subjektivem Bedarf nach schnellerem Wirkeintritt als nach der Einnahme von einer Extradosis einer schnell resorbierbaren und löslichen Levodopa Darreichungsform. Einige an Off-Phasen gekoppelte Phänomene, die erfahrungsgemäß schwer mit Umstellungen des oralen Therapieregimes zu beeinflussen sind, konnten mit subkutanem Apomorphin angegangen werden. Dazu gehören dystone Verkrampfungen und sensorische Phäno- 
mene wie Schmerzen [12-14], Dysphagie [15-17], Blasenentleerungs- und Defäkationsstörungen [18, 19], Panik und suizidale Einengung [20].

Apomorphin wurde schon im 19. Jahrhundert zur Behandlung des Tremors oral eingesetzt [21]. Von allen Dopaminagonisten kommt es in seiner klinischen Wirkung derjenigen von Levodopa am nächsten, wirkt allerdings kaum, wenn oral verabreicht. Apomorphin ist ein gemischter D1und D2-Agonist. Apomorphin ist zugelassen „zur Behandlung von Parkinson-Patienten mit behindernden motorischen Komplikationen (On-Off Phänomene), die trotz individuell eingestellter Behandlung mit Levodopa (mit einem peripherem Decarboxylase-hemmer) und/oder anderen Dopaminagonisten weiter bestehen “ [22].

Die Wirksamkeit der sukutanen Apomorphin-Infusion wurde in vielen nicht verblindeten, nicht placebokontrollierten Studien seit Ende der 1970er-Jahren belegt: Die Therapie reduzierte die Off-Phasen um etwa $60 \%$ und die Dyskinesien um nahezu 50\% [23-25]. In der großen multizentrischen doppelblinden placebokontrollierten Studie mit 107 Patienten, reduzierte die subkutane Apomorphin-Infusion tagsüber (mittlere Dosis $4,68 \mathrm{mg} / \mathrm{h}$, 3-8 mg/h) die Off-Zeit im Vergleich zu einer Placebo-Infusion signifikant: $2,47 \mathrm{~h}$ pro Tag in der Apomorphin-Gruppe gegenüber $-0,58 \mathrm{~h}$ pro Tag in der Placebo-Gruppe $p=0,0025$ [26]. In dieser Studie wurde Apomorphin ohne unerwartete Nebenwirkungen gut vertragen. Bemerkenswerterweise für einen Dopaminagonisten traten keine vermehrten neuropsychiatrischen unerwünschten Wirkungen gegenüber Placebo auf. Visuelle Halluzinationen sowie Impulskontrollstörungen werden nicht als Kontraindikation für die Apomorphin-Pumpe bei Patienten gesehen, die unter oralen Dopaminagonisten diese Probleme entwickeln [27-29].

Eine Vorbehandlung mit dem extrazerebralen Dopaminantagonisten Domperidon $3 \times 10 \mathrm{mg}$ (Filmtabletten oder Suspension) über mindestens 24 Stunden vor einer ersten Apomorphin-Gabe wird zur Vermeidung von peripheren dopaminergen Nebenwirkungen (Übelkeit, Erbrechen, Blutdruckabfall, Müdigkeit) über 3-7 Tage eingesetzt [30]. Diese habituieren häufig, sodass im weiteren Verlauf Domperidon aufgegeben werden kann. Zu berücksichtigen ist, dass die Domperidon-Zulassung mit Reduktion der maximalen Tagesdosis auf $30 \mathrm{mg}$ und Dauer der Behandlung auf eine Woche eingeschränkt wurde. Epidemiologische Studien wiesen einen Zusammenhang zwischen der Einnahme Domperidon und einem erhöhten Risiko von schwerwiegenden ventrikulären Arrhythmien oder plötzlichem Herztod auf. Für Parkinson-Patienten gibt es zu Domperidon jedoch keine Alternative als Antiemetikum und es wird europaweit weiter eingesetzt. Nach einer Studie bei 5114 Parkinson-Patienten war die chronische Einnahme von Domperidon mit einem 2-fach erhöhten Risiko von Mortalität jedweder Ursache im Vergleich zu Parkinson-Patienten verbunden, die

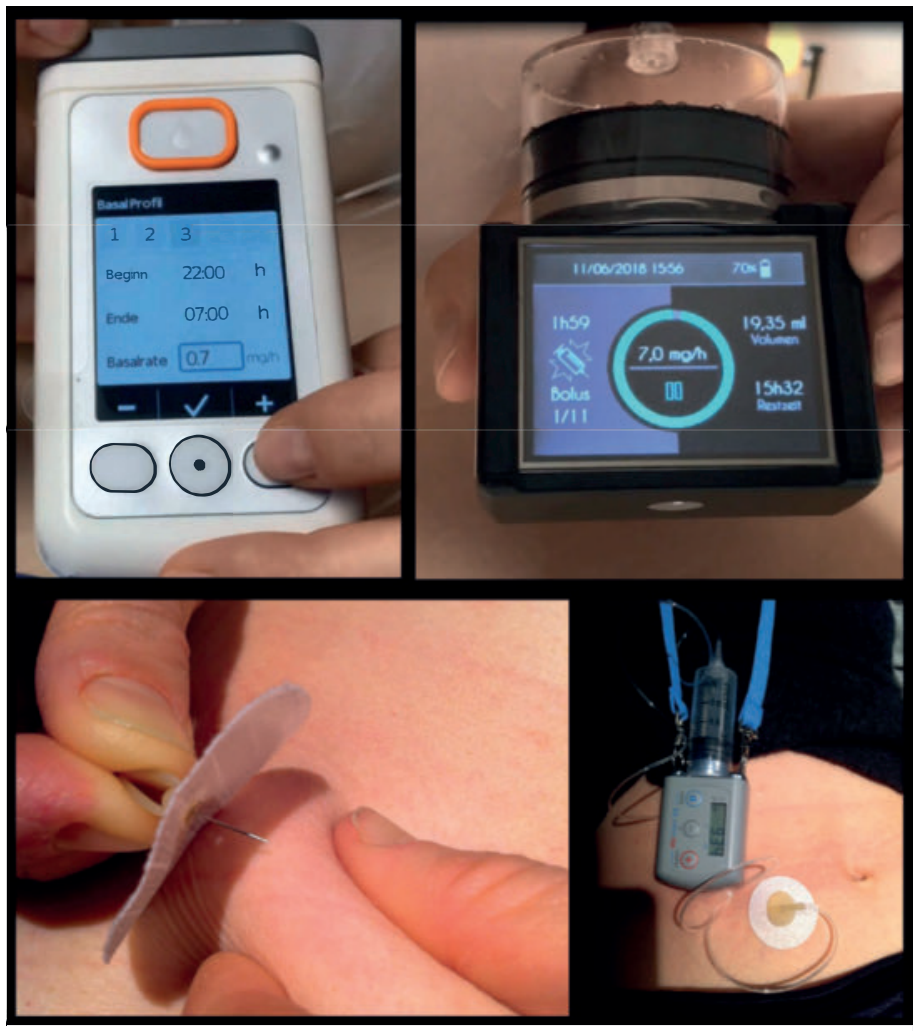

- Abb. 1 Apomorphin-Pumpen und „Reißzweckenkatheter” (Stahl- oder Kunststoffkanülen können verwendet werden) für die subkutane Infusion. Quelle: ๑ A. Ceballos-Baumann, München

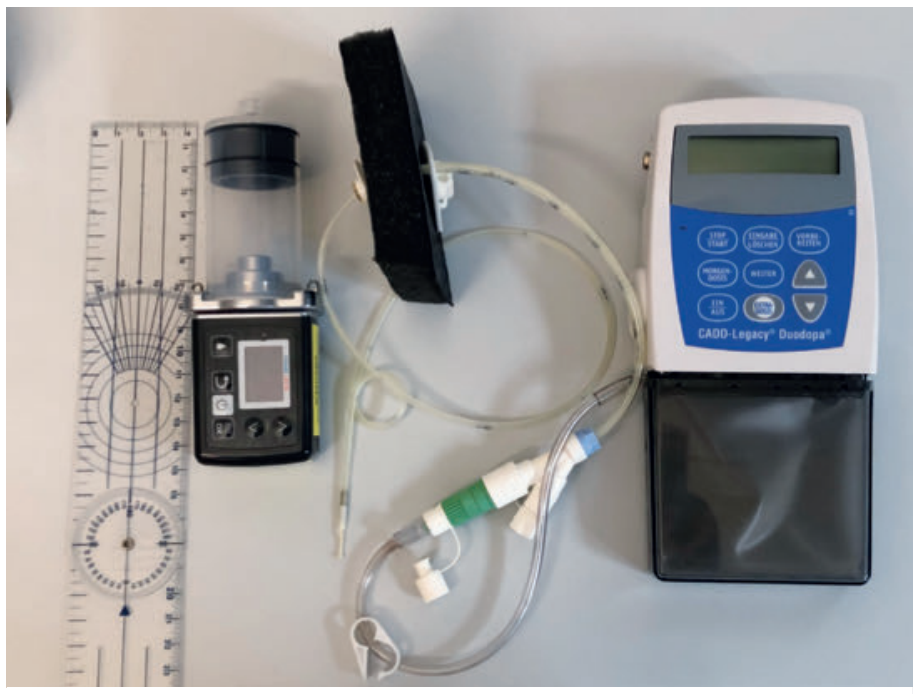

- Abb. 2 Links die Crono LECIG (CE 0476) für die Infusion in das Jejunum von Levodopa/Entacapon/Carbidopa intestinales Gel (LECIG) und rechts die CADD-Legacy 1400-Pumpe (CE 0473) für die Infusion in das Jejunum von Levodopa/Carbidopa intestinales GEL (LCIG) mit der äußeren Transabdominalsonde (gastraler Schenkel) und einer feinlumigeren inneren Applikationssonde (jejunaler Schenkel) für die PEG (schwarzes Schaumstoffstück soll die Bauchwand darstellen). Quelle: $\odot$ A. CeballosBaumann, München 


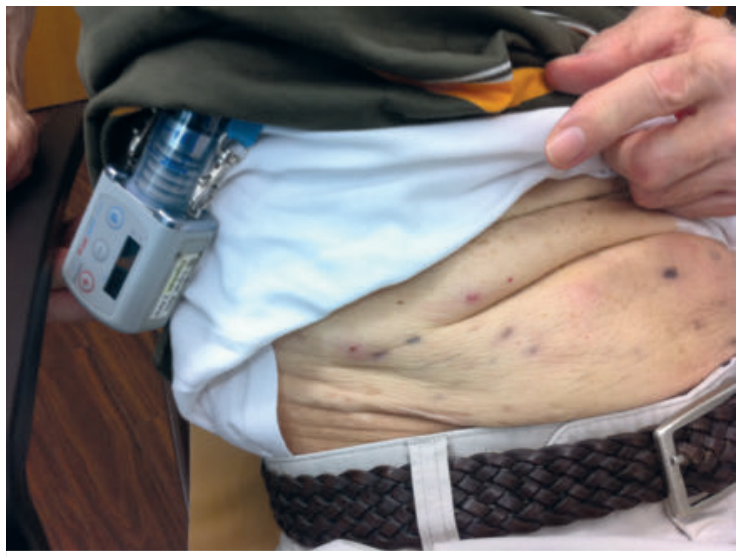

- Abb. 3 Insgesamt bilden 70-80\% der Patienten Noduli (subkutane Granulome) aus, die sich in der Regel zurückbilden. Die Größe dieser Knötchen hängt von der Konzentration, Kontaktzeit, Infusionstiefe und der Menge der Apomorphin-Lösung ab. Quelle: (c) A. Ceballos-Baumann, München

nie Domperidon eingenommen hatten. Das Risiko war im ersten Monat der Anwendung am höchsten [31].

Die Apomorphin-Pumpe läuft in der Regel nur während der Wachzeit. Bei manchen Patienten hat sich eine 24-stündige Applikation bewährt, wenn der Patient über andersartig nicht behebbare Symptome während der Nachtstunden klagt. Vereinzelte Patienten tragen sie nur in der Nacht. Die subkutane Infusionsstelle sollte möglichst jeden Tag gewechselt werden. Die kontinuierliche Infusion sollte mit einer Rate von 0,5-1 mg Apomorphin pro Stunde am ersten Tag begonnen werden, danach wird nach individueller Besserung die Dosis weiter erhöht. Dabei sollte eine Erhöhung der Infusionsrate um 0,5 mg pro Stunde aber nicht überschritten und zwischen diesen Dosiserhöhungen eine Zeitspanne von mindestens 4 Stunden eingehalten werden. Stündliche Infusionsraten liegen typischerweise im Bereich zwischen $4 \mathrm{mg}$ und $7 \mathrm{mg}$ und laufen meist ca. 16 Stunden. Die meisten Patienten benötigen als Ergänzung zur kontinuierlichen Infusion zusätzlich einen Bolus mittels des Pumpsystems. Zur Vereinfachung der oralen Therapie und zur Vermeidung additiver unerwünschter Arzneimittelwirkungen sowie -interaktionen sollte mittelfristig versucht werden, die oralen Antiparkinsonika auf wenige zusätzliche Gaben von Levodopa oder ganz auf die Monotherapie mit Apomorphin während der Pumpenlaufzeit umzustellen. Letzteres gelingt aber in der Routineversorgung selten. Für die meisten Patienten mit der Apomorphin-Pumpe besteht die Notwendigkeit auch während der Laufzeit der Infusion weiterhin Levodopa oral einzunehmen. Daher besteht großes Interesse für die laufenden klinischen Prüfungen von Levodopa/Carbidopa-Darreichungen für die subkutane Infusion.

Als unerwünschte Effekte der subkutanen Verabreichung von Apomorphin werden Gähnen, Übelkeit und eine or- thostatische Hypotonie trotz chronischer DomperidonGabe beobachtet. Ferner kommt es zu Müdigkeit und der Verstärkung von Dyskinesien vor allem am Anfang der Einstellung, wenn Levodopa noch nicht reduziert wurde. Psychosen, Tränenfluss, Hypersexualität und herabgesetzte Libido wurden beschrieben [23-25, 32]. 70-80\% der Patienten bilden Noduli (subkutane Granulome unter der Haut, \ Abb. 3) eine Form der Pannikulitis, eine örtliche, entzündliche Reaktion des Unterhautgewebes, selten bilden sich kutane Abszesse. Die Hautnoduli hängen von der Konzentration, Kontaktzeit, Infusionstiefe, immunologische Reaktion zu einem der Inhaltsstoffe, der Verdünnung und der Menge der Apomorphinlösung ab [33]. Sie können die gleichmäßige Apomorphin-Resorption stören. Als Therapie kommen Massagen, Salben sowie Silicon-Pflaster in Betracht. Wechsel der Injektionsstellen, eine trockene Punktion (kein Tropfen an der Nadel) sowie Hygiene sind nach pflegerischer Erfahrung wichtig, um das Problem von Beginn an zu minimieren.

\section{Jejunale Levodopa-Carbidopa und Levodopa-Entacapon-Carbidopa- intestinales Gel (LCIG, LECIG)-Infusion}

Dass kontinuierliche duodenale/jejunale Infusionen von Levodopa und Dekarboxylasehemmer wie dem Carbidopa über eine Umgehung der gestörten Magenentleerung Wirkungsfluktuationen glätten und Dyskinesien reduzieren können, ist schon in den 1980er-Jahren beschrieben worden [34]. Die direkte Infusion in die Region des proximalen Dünndarms führte zu einem gleichmäßigen Levodopaspiegel im Blut. Aber erst durch den pharmakokinetischen Kunstgriff in Matrixform gebundenes Levodopa mit Carbidopa in ein mit wenig Flüssigkeit auskommendes und damit Volumen sparendes Gel produzieren zu können, war es möglich, dieses Konzept in die Klinik einzuführen. LCIG wurde Mitte der 2000er-Jahre zugelassen. 2021 folgte die Markteinführung eines weiteren Levodopa-Carbidopa Gels mit Entacapon (LECIG) ebenso zur intestinalen Anwendung als „Behandlung der fortgeschrittenen Parkinson-Krankheit mit schweren motorischen Fluktuationen und Hyperkinesien oder Dyskinesien, wenn verfügbare orale Kombinationen von Parkinson-Arzneimitteln nicht zu zufriedenstellenden Behandlungsergebnissen geführt haben“. Die jejunale Infusion von LCIG und LECIG mittels einer perkutanen endoskopischen Gastrostomie (PEG) in das Jejunum über eine 2-lumige Sonde, einer äußeren Transabdominalsonde (gastraler Schenkel) und einer feinlumigeren inneren Applikationssonde (jejunaler Schenkel) erfordert die Zusammenarbeit mit erfahrenen Gastroenterologen. Das Ende der Applikationssonde sollte durch den Magen und Duodenum nach dem Treitzschen Band in das proximale Jejunum geführt werden. Um die Indikation abzusichern, kann vor der PEG in einer Testphase über eine Nasensonde, die unter Röntgendurchleuchtung oder endoskopisch bis jenseits des Treitzschen Bandes über den Magen und 
Duodenum hinaus vorgeschoben wurde, die Wirksamkeit der Therapie überprüft werden.

In einer doppelblinden multizentrischen Studie wurden 71 Parkinson-Patienten im fortgeschrittenen Stadium in 2 Gruppen randomisiert, nachdem man ihnen eine PEG mit jejunaler Sonde gelegt hatte [35]. Entweder bekamen sie das LCIG jejunal in Kombination mit oralem Placebo oder ein Placebo-Gel über die Sonde und Levodopa/Carbidopa konventionell oral. Primäres Ziel war die Veränderung der motorischen Off-Zeit nach einer insgesamt 4-wöchigen Titrationsphase mit einer sich anschließenden Nachbeobachtungszeit von 8 Wochen. Die motorische Off-Zeit verringerte sich in der Verum-Gruppe um durchschnittlich 4,04 Stunden pro Tag; in der Placebo-Gruppe um 2,14 Stunden pro Tag. Die On-Zeit ohne beeinträchtigende Dyskinesien nahm unter der Levodopa-Geltherapie im Mittel um 4,11 Stunden zu, bei konventioneller oraler Verabreichung um 2,24 Stunden. Mit der Verkürzung der Off-Zeit und Reduktion verknüpft war eine signifikante Verbesserung der Lebensqualität. Ein geringerer Care Giver Burden konnte nicht nachgewiesen werden. Bei der PEG-Anlage mit der jejunalen Sonde kam es bei 12 der 71 Patienten zu schwerwiegenden Ereignissen wie z. B. Peritonitis. Die Studie ging über 3 Monate. Verlaufsbeobachtungen von im Mittel 3 Jahren sprechen für einen anhaltenden Effekt der jejunalen LCIG-Pumpe [36, 37]. In einer Studie mit 72 Patienten über einem mittleren Beobachtungszeitraum von 22 (max. 48) Monaten wird weiterhin eine nachhaltige Glättung der Wirkungsschwankungen bei signifikanter Zunahme an Dyskinesien berichtet [38]. In 28 Patienten wurde in diesem Zeitraum die Levodopa/Carbidopa-Pumpentherapie abgebrochen, bei 13 Patienten wegen unbefriedigender, bei 12 wegen unerwünschter Wirkungen (4 davon PEG-, sonden- und pumpenassoziiert) und bei 4 wegen mangelnder Patientenakzeptanz.

Die Indikationen und das Vorgehen beider Darreichungsformen LCIG und LECIG sind gleich bis auf die verwendete Pumpe und die Dosierung von Levodopa. Nach den Studien wird die gleiche Morgendosis an Levodopa-Äquivalenz gewählt, bei den bedarfsorientierten Extradosen (Bolus) und der Flussrate sollte bei LECIG mit einem Drittel weniger Levodopa in Bezug auf die orale oder intestinale Darreichung ohne Entacapon gerechnet werden [39-41]. Denn Entacapon als Hemmer der Catechol-O-Methyltransferase führt neben dem Decarboylasehemmer Carbidopa zu einer weiteren Reduktion der Metabolisierung von Levodopa und zu einer Steigerung der Bioverfügbarkeit von Levodopa um 34\% [41, 42]. Durch die gesteigerte Bioverfügbarkeit von Levodopa bei LECIG braucht man weniger an Infusionsvolumen als ohne Entacapon und kann eine kleinere und geräuschärmere Pumpe verwenden als bei LCIG. Nur die CADD-Legacy 1400-Pumpe darf für die enterale Infusion von LCIG sowie die Pumpe CRONO LECIG nur für die enterale Verabreichung von LECIG herangezogen wer- den ( $\mathbf{A}$ bb. 2). In einer Verlaufsbeobachtung mit 24 Patienten über 304 Tage (Median) musste die Therapie mit LECIG bei einem Patienten wegen Halluzinationen und bei 3 Patienten wegen Diarrhoen abgebrochen werden [39]. Entacapon ist häufig mit Diarrhoen und immer mit der an sich harmlosen Gelbverfärbung des Urins verbunden. Patienten, die unter oralem Entacapon oder Tolcapon Diarrhoen entwickelt hatten, sollten nicht für eine Pumpe mit LECIG vorgesehen werden.

Ein plötzlicher Wirkungsverlust der jejunalen Infusionstherapie spricht für eine Abklemmung, eine Dislokation des Endes der Applikationssonde nach distal im Jejunum oder proximal zum Magen, eine Diskonnektion oder einer Verstopfung des feinlumigen jejunalen Schenkels in der PEG-Sonde. Dies muss zu einer raschen Überprüfung in einem entsprechend ausgewiesenen Zentrum führen. Ferner sind die allgemeinen Komplikationen einer PEG wie Wundinfektion bis hin zur Magen-/Darmperforation, Peritonitis und Malnutrition zu beachten. Seit 2009 mehren sich Berichte zu schwerwiegenden, teilweise GuillainBarré-ähnlichen Neuropathien unter der jejunalen Levodopa-Infusion. Die genaue Pathophysiologie dieser Neuropathie ist noch nicht bekannt. Es werden autoimmunologische Faktoren sowie ein relativer Vitamin- $B_{12}$ - und $-B_{6}$ - Mangel ursächlich diskutiert. Es treten jedoch Fälle auf trotz einer Supplementierung mit Vitamin $B_{12}[43,44]$.

\section{THS („Hirnschrittmacher“)}

Die THS ist eine anerkannte und nicht nur für die Parkinsontherapie zugelassene geräteunterstützte Therapie, bei dem durch implantierte Elektroden umschriebenes Hirngewebe präzise ständig hochfrequent stimuliert wird (für eine detailliertere Darstellung siehe die 2 Artikel in dieser Ausgabe der Nervenheilkunde) [45, 46]. Die THS wurde in den 1990er-Jahren zur Behandlung der fortgeschrittenen Parkinson-Krankheit eingeführt mit Implantation der Elektroden in den Nucleus subthalamicus, nachdem die Überaktivität dieses Kerns als ein grundlegender Befund bei der Parkinsonakinese feststand [47]. Die Stimulationssysteme werden unter die Haut implantiert und bestehen aus den stimulierenden Hirnelektroden, Verbindungskabel und dem Impulsgeber mit Batterie. Seit einigen Jahren gibt es durch Induktion wieder aufladbare Impulsgeber mit Batterie. Ansonsten ist abhängig von den Stimulationsparametern nach 2-7 Jahren ein Batteriewechsel fällig. Die Stimulationsparameter wie Pulsbreite, Spannung bzw. Stromstärke, Frequenz der Stimulation können über Telemetrie verändert werden. Die Ausbreitung des Stroms kann bei bestimmten Systemen durch eine horizontale Segmentierung der mittleren beiden Ringelektroden in jeweils 3 selektiv steuerbare Kontakte präziser fokussiert werden (direktionale Stimulation). Dieser Ansatz wurde in einer multizentrischen Studie mit aufladbarem Impulsgeber untersucht [48]. Das macht die Programmierung zwar zeitaufwendig, aber das zu stimulierende Gewebe kann mit 
bis zu 8 aktiven Kontakten pro Elektrode postoperativ und im Verlauf angepasst werden, um unerwünschte Effekte wie z. B. die Mitstimulation von Fasern der Capsula interna zu minimieren. Der Nucleus subthalamicus (STN) hat sich über die letzten Jahrzehnte als der geeignetste Zielpunkt für die Stimulation herausgestellt. Hier werden die akinetisch-rigide Symptomatik mit einem levodopaähnlichen Effekt sowie der Tremor positiv beeinflusst. Die beiden anderen Zielpunkte, der Globus pallidus internus (GPi) und der Nucleus ventralis intermedius (VIM), werden beim IPS nur noch ausnahmsweise für Spezialindikationen im Rahmen des IPS angegangen wie z. B. schwerer Aktionstremor (VIM) oder Dyskinesien (GPi).

Um die Elektroden millimetergenau an den Zielpunkt zu bringen, wo sie hingehören, wird ein stereotaktischer Rahmen (3-dimensionales Ringsystem) am Schädel des Patienten befestigt. Der Zielpunkt wird mithilfe von Kernspintomografie und/oder Fusion mit einer Röntgen-Computertomografie des Gehirns sowie spezieller Planungssoftware berechnet. Die Operationen finden größtenteils bei vollem Bewusstsein statt. Der Patient kann so bei den Teststimulationen zur optimalen Lokalisation des Zielpunktes beitragen, indem er über unerwünschte Wirkungen wie Missempfindungen befragt und die Wirkung auf Akinese, Tremor und Rigor evaluiert werden kann. Die Elektroden werden mit Mikrometerschrauben über kleinste Bohrlöcher am Schädel zum berechneten Zielpunkt langsam geführt. Nach Sicherung der implantierten Elektroden am Schädel wird der Impulsgeber wie ein Herzschrittmacher meist unter dem Schlüsselbein implantiert und mit einem unter der Haut vorgeschobenen Kabel mit den Elektroden am Schädel verbunden. Dieser Schritt erfolgt in der Regel in einer kurzen Vollnarkose. Es kann zu Komplikationen, wie Blutungen im Gehirn, Austritt von Liquor oder Infektionen und Dysfunktion des implantierten Systems kommen $[45,46]$. Bestimmte Voruntersuchungen werden gefordert: eine Kernspintomografie (MRT) des Gehirns, eine neuropsychologische und neuropsychiatrische Untersuchung (z. B. Ausschluss Demenz, Abklärung zu Impulskontrollstörungen), Dokumentation des Ansprechens des Patienten auf Levodopa und Ausschluss anderer gegen eine STN-Stimulation sprechende Störungen (detailliertere Darstellung in 2 Artikel dieser Ausgabe $[45,46])$.

\section{Differenzialindikation und Entscheidung für eine GUT}

Bisher gibt es keine randomisierten kontrollierten Studien, die direkt subkutane Apomorphin-Pumpe, die jejunalen LCIG-/LECIG-Pumpen und die THS miteinander vergleichen. Klinische Erfahrung und Studien legen nahe, dass THS, LCIG/LECIG und Apo-Pumpe vergleichbar sind in der Reduktion von Off-Zeit [5, 49]. Die Wahl basiert auf Faktoren wie Verfügbarkeit, Wirtschaftlichkeit, lokale Expertise, Arztvorlieben sowie der Präferenzen und Ängste der Pati- enten und ihrer Bezugspersonen. In einer Beobachtungsstudie, in die Patienten mit 101 STN-THS ( $n=101)$, jejunale LCIG-Pumpe ( $n=33$ ) und subkutane Apomorphin-Pumpe $(n=39)$ aufgenommen werden konnten, verbesserten sich in allen Gruppen der krankheitsspezifische Lebensqualität-Score PDQ-8 und der nicht Motorische-Symptome Gesamt-Score bei Nachbeobachtung. Die tägliche Levodopa-Äquivalenzdosis war nur nach STN-THS signifikant reduziert. Darüber hinaus ergaben sich unterschiedliche Profile für die nicht motorischen Symptome: STN-DBS verbesserte die Harn-/Sexualfunktion, Stimmung/Kognition und Schlaf/Müdigkeit. LCIG-Infusionen verbesserte alle 3 letzteren Domänen und zusätzlich gastrointestinale Symptome, während die Apomorphin-Pumpe die Domänen Stimmung/Kognition, Wahrnehmungsprobleme/Halluzinationen sowie Aufmerksamkeit/Gedächtnis positiv beeinflusste [50]. In der S3 Leitlinie wird die bessere Datenlage für den Einsatz der STN-THS im Vergleich zur bestmöglichen medikamentösen Therapie gegenüber den beiden Medikamentenpumpen hervorgehoben [4]. Daher wird für den Einsatz der STN-THS von „sollte angeboten werden“ geschrieben, während bei den Pumpen die „kann“ Formulierung gewählt wird.

Aspekte wie eine bessere Datenlage können bei dem Entscheidungsprozess mit dem Patienten erörtert werden. Nach einer niederländischen Studie zogen es über $90 \%$ der Patienten vor, an der Entscheidungsfindung beteiligt zu sein, welche der 3 GUT angewendet werden soll. Bei etwa der Hälfte der Befragten (47\%) entsprach ihre bevorzugte Rolle nicht ihrer erfahrenen Rolle; $28 \%$ hatten eine aktivere Rolle, als sie es sich gewünscht hätten. Obwohl sich $77 \%$ zum Zeitpunkt der Entscheidung vollständig informiert vorkamen, gaben nur $41 \%$ an, alle 3 Therapieoptionen zu kennen [51]. Jüngere Patienten profitieren im Hinblickt auf Lebensqualität von der THS mehr als ältere. Bei jungen Patienten mit einem aktiven Lebensstil gibt es überdies meist Vorbehalte gegenüber den Pumpenverfahren aufgrund des Stigmas einer externen Pumpe und des pflegerischen Aufwands. Höheres Alter allein sollte kein entscheidender Faktor gegen die Indikation zur THS mehr sein, wenn der Fokus streng auf Patienten mit schwer kontrollierbaren On-Off-Fluktuationen und erhaltener Kognition gehalten wird [52]. Spezifische Kontraindikationen für die THS sind Demenz, ernste psychische und somatische Erkrankungen, neurochirurgische Kontraindikationen sowie Hirnatrophie. Mit einer Besserung von schlecht auf Levodopa ansprechenden Symptomen wie Stimm-, Sprech-, Schluckstörungen und motorischen Blockaden (Freezing) ist nicht zu rechnen (Kasten „Kontraindikationen“). Diese Symptome können sich sogar nach der THS verschlechtern. 


\section{TIEFE HIRNSTIMULATION}

\section{Spezifische Kontraindikationen}

- Demenz

- psychiatrische Vorerkrankungen (Impulskontrollstörungen und Halluzinationen können sich durch die Reduktion der Dopaminagonisten postoperativ verbessern)

- ernste somatische Erkrankungen

- Hirnatrophie

- unrealistische Erwartungen wie Besserung von schlecht auf Levodopa ansprechende Symptome wie Gleichgewichts-, Stimm-, Sprech-, Schluckstörungen und motorische Blockaden (Freezing)

Bei vielen Patienten bestehen Kontraindikationen für die THS und/oder können sich für dieses Verfahren nicht entscheiden. Die Einhaltung von 5-10 Einnahmezeiten von Levodopa mit Berücksichtigung von Karenzzeiten vor und nach den Mahlzeiten ist für viele Patienten schwer zu organisieren. In dieser Situation kann eine Pumpentherapie mit Anlegen morgens und Abnehmen zur Nacht für Patienten besonders hilfreich sein. Jejunale Levodopa oder subkutane Apomorphin-Pumpen können für Patienten im Alter von über 70 Jahren in Betracht gezogen werden, die leichte oder mittelschwere kognitive Beeinträchtigungen, schwere Depressionen oder andere Kontraindikationen für DBS haben. Stürze sind mit kognitiver Dysfunktion verbunden und werden allerdings mit GUT wahrscheinlich häufiger auftreten [5, 49]. Bei idealen Kandidaten für die THS (z. B. um die 60 Jahre alt, ansonsten gesund, kognitiv fit, in On Phase eine gute Wirkung von Levodopa, keine Symptome beim Sprechen, keine Gleichgewichtstörungen, kein Freezing beim Gehen, aber eindeutige und störende Levodopawirkungsschwankungen mit Offs und Dyskinesien) kann die Angst vor dem Risiko einer Hirnoperation oder die Abneigung einer „Prothese im Hirn“ überwiegen, sodass sie eher einem Pumpenverfahren zuneigen. Für Patienten in Ambivalenz vor einer THS bietet sich die unverbindliche Testbarkeit der Apomorphin-Pumpe ohne Notwendigkeit eines operativen Eingriffs als Übergangslösung an, zumal perioperativ der Einsatz der Apomorphin-Pumpe hilfreich ist [53-55]. Andererseits kann die Wirksamkeit einer jejunalen Infusion eines Levodopa/Carbidopa-Gels, bei der auf orale Antiparkinsonika während der Pumpenlaufzeit verzichtet werden kann, die Risiken und Strapazen einer PEG sowie einer größeren Pumpe aufwiegen.

Eine operative Behandlung wie die THS oder das Tragen von Medikamentenpumpen bleibt immer eine individuelle Entscheidung, bei der es viel abzuwägen gilt. Geeignete Patienten sollten über alle 3 GUT gut informiert werden. Idealerweise sollte ein „Shared Decision Making“ als operationalisiertes Konzept der Patientenbeteiligung gemeinsam, mit den Patienten und seinen Bezugspersonen zum Zuge kommen in Zentren, die über Erfahrung in - und Zugang zu - allen Therapieformen verfügen [5, $49,53]$.

\section{FAZIT FÜR DIE PRAXIS}

- Die geräteunterstützten Therapien mittels äußerlich tragbarer Pumpen für Apomorphin subkutan und Levodopa/Carbidopa oder Levodopa/Entacapon/Carbidopa intestinales Gel via einer PEG mit jejunaler Applikationsonde und THS sind ressourcenintensive und eine unterschiedliche Logistik erfordernde Therapien der Parkinson-Krankheit mit Wirkfluktuationen von Levodopa (On-Off, Dyskinesien).

- Die 5-2-1-Kriterien als Screening für den Einsatz von geräteunterstützten Therapien: mehr als 5 Einnahmezeitpunkte von Levodopa pro Tag, mehr als 2 Stunden pro Tag im Off, mehr als 1 Stunde störende Dyskinesien.

- Die Apomorphin-Pumpe subkutan kann ohne invasiven Eingriff ausprobiert werden.

- Patienten mit jejunalen Pumpen für LevodopaCarbidopa oder Levodopa-Entacapon-Carbidopa intestinales Gel via einer PEG brauchen während der Laufzeit der Pumpe keine orale ParkinsonMedikation im Gegensatz zu den meisten Patienten mit der Apormorphin-Pumpe und der THS.

- Der ideale Parkinson-Patient für die THS ist um die 60 Jahre alt, ansonsten gesund, kognitiv und psychiatrisch unauffällig, weist ein normales Schädel-MRT auf, hat im On keine Symptome beim Sprechen, Stimme, Gleichgewicht und keine motorischen Gangblockaden (Freezing), aber eindeutige und störende Wirkungsschwankungen mit Offs und Dyskinesien, obwohl die Tagesdosis von Levodopa schon auf mehr als 5 Einnahmezeitpunkte fraktioniert wurde.

Interessenkonflikt

Erklärung zu finanziellen Interessen

Forschungsförderung erhalten: nein; Honorar/geldwerten Vorteil für Referententätigkeit erhalten: ja; Bezahlter Berater/interner Schulungsreferent/Gehaltsempfänger: ja; Patent/Geschäftsanteile/Aktien (Autor/Partner, Ehepartner, Kinder) an Firma (Nicht-Sponsor der Veranstaltung): nein; Patent/Geschäftsanteile/Aktien (Autor/Partner, Ehepartner, Kinder) an Firma (Sponsor der Veranstaltung): nein.

Erklärung zu nicht finanziellen Interessen

Der Autor gibt an, dass kein Interessenkonflikt besteht. 


\section{Korrespondenzadresse}

Prof. Dr. med. Andrés Ceballos-Baumann

Abt. für Neurologie und klinische Neurophysiologie mit Parkinson-Fachklinik

Schön Klinik München Schwabing

Parzivalplatz 4, 80804 München, Deutschland

Tel. 089/36087131

aceballos-baumann@schoen-klinik.de

\section{Literatur}

[1] Politis M, Wu K, Molloy S, et al. Parkinson's disease symptoms: the patient's perspective. Mov Disord 2010; 25(11): 1646-51

[2] Schrag A, Quinn N. Dyskinesias and motor fluctuations in Parkinson's disease. A community-based study. Brain 2000; 123 (Pt 11): 2297-305

[3] Schuepbach WM, Rau J, Knudsen K, et al. Neurostimulation for Parkinson's disease with early motor complications. N Engl J Med 2013; 368(7): 610-22

[4] DGN Ld. Idiopathisches Parkinson-Syndrom, Entwicklungsstufe: S3, Stand: 1. Januar 2016, Gültig bis: 31. Dezember 2020, AWMF-Registernummer: 030/010. https://dgnorg/ leitlinien/030-010-idiopathisches-parkinson-syndrom/. 2016

[5] Odin P, Ray Chaudhuri K, et al. Collective physician perspectives on non-oral medication approaches for the management of clinically relevant unresolved issues in Parkinson's disease: Consensus from an international survey and discussion program. Parkinsonism Relat Disord 2015; 21(10): 1133-44

[6] Williams DR, Evans AH, Fung VSC, et al. Practical approaches to commencing device-assisted therapies for Parkinson disease in Australia. Intern Med J 2017; 47(10): 1107-13

[7] Norlin JM, Willis M, Persson U, et al. Swedish guidelines for device-aided therapies in Parkinson's disease -Economic evaluation and implementation. Acta Neurol Scand 2021; 144(2): 170-8

[8] Santos-Garcia D, de Deus Fonticoba T, Suarez Castro J, et al. 5-2-1 Criteria: A Simple Screening Tool for Identifying Advanced PD Patients Who Need an Optimization of Parkinson's Treatment. Parkinsons Dis 2020; 2020: 7537924

[9] Aldred J, Anca-Herschkovitsch M, Antonini A, et al. Application of the ' $5-2-1$ ' screening criteria in advanced Parkinson's disease: interim analysis of DUOGLOBE. Neurodegener Dis Manag 2020; 10(5): 309-23

[10] Smilowska K, van Wamelen DJ, Pietrzykowski T, et al. Cost-Effectiveness of Device-Aided Therapies in Parkinson's Disease: A Structured Review. J Parkinsons Dis 2021; 11(2): 475-89

[11] Marsili L, Bologna M, Miyasaki JM, et al. Parkinson's disease advanced therapies - A systematic review: More unanswered questions than guidance. Parkinsonism Relat Disord 2020, ePub

[12] Factor SA, Brown DL, Molho ES. Subcutaneous apomorphine injections as a treatment for intractable pain in Parkinson's disease. Mov Disord 2000; 15(1): 167-9

[13] Reuter I, Ellis CM, Ray Chaudhuri K. Nocturnal subcutaneous apomorphine infusion in Parkinson's disease and restless legs syndrome. Acta Neurol Scand 1999; 100(3): 163-7
[14] Lefaucheur R, Berthelot L, Senant J, et al. Acute genital pain during non-motor fluctuations improved by apomorphine. Mov Disord 2013; 28(5): 687-8

[15] Tison F, Wiart L, Guatterie M, et al. Effects of central dopaminergic stimulation by apomorphine on swallowing disorders in Parkinson's disease. Mov Disord 1996; 11(6): 729-32

[16] Kempster PA, Lees AJ, Crichton P, et al. Off-period belching due to a reversible disturbance of oesophageal motility in Parkinson's disease and its treatment with apomorphine. Mov Disord 1989; 4(1): 47-52

[17] Bailbe M, Bataille B, Paquereau ], et al. [Improvement in swallowing difficulties treated by subcutaneous apomorphine infusion after deep brain stimulation in Parkinson's disease]. Rev Neurol 2004; 160(3): 352-3

[18] Christmas TJ, Kempster PA, Chapple CR, et al. Role of subcutaneous apomorphine in parkinsonian voiding dysfunction. Lancet 1988; 2(8626-8627): 1451-3

[19] Edwards LL, Quigley EM, Harned RK, et al. Defecatory function in Parkinson's disease: response to apomorphine. Ann Neurol 1993; 33(5): 490-3

[20] Di Rosa AE, Epifanio A, Antonini A, et al. Continuous apomorphine infusion and neuropsychiatric disorders: a controlled study in patients with advanced Parkinson's disease. Neurol Sci 2003; 24(3): 174-5

[21] Weil. D l'apomorphine dans certain troubles nerveux. Lyon Med 1884; 48: 411-9

[22] Tonges L, Ceballos-Baumann A, Honig H, et al. [Practical use of continuous apomorphine infusion via pump]. Fortschr Neurol Psychiatr 2017; 85(9): 516-35

[23] Corsini GU, Del Zompo M, Gessa GL, et al. Therapeutic efficacy of apomorphine combined with an extracerebral inhibitor of dopamine receptors in Parkinson's disease. Lancet 1979; 1(8123): 954-6

[24] Stibe C, Lees AJ, Kempster PA, et al. Subcutaneous apomorphine in Parkinsonian on-off oscillations. Lancet 1988; 1(8582): 403-6

[25] Garcia Ruiz PJ, Sesar Ignacio A, et al. Efficacy of long-term continuous subcutaneous apomorphine infusion in advanced Parkinson's disease with motor fluctuations: a multicenter study. Mov Disord 2008; 23(8): 1130-6

[26] Katzenschlager R, Poewe W, Rascol O, et al. Apomorphine subcutaneous infusion in patients with Parkinson's disease with persistent motor fluctuations (TOLEDO): a multicentre, double-blind, randomised, placebo-controlled trial. Lancet Neurol 2018; 17(9): 749-59

[27] Borgemeester RW, Lees A], van Laar T. Parkinson's disease, visual hallucinations and apomorphine: A review of the available evidence. Parkinsonism Relat Disord 2016; 27: 35-40

[28] Todorova A, Samuel M, Brown RG, et al. Infusion Therapies and Development of Impulse Control Disorders in Advanced Parkinson Disease: Clinical Experience After 3 Years' Follow-up. Clin Neuropharmacol 2015; 38(4): 132-4

[29] Barbosa P, Lees A], Magee C, et al. A Retrospective Evaluation of the Frequency of Impulsive Compulsive Behaviors in Parkinson's Disease Patients Treated with Continuous Waking Day Apomorphine Pumps. Mov Disord Clin Pract 2017; 4(3): 323-8

[30] Trenkwalder C, Chaudhuri KR, Garcia Ruiz P], et al. Expert Consensus Group report on the use of apomorphine in the treatment of Parkinson's disease - Clinical practice recommendations. Parkinsonism Relat Disord 2015; 21(9): 1023-30

[31] Simeonova M, de Vries F, Pouwels S, et al. Increased risk of all-cause mortality associated with domperidone use in 
Parkinson's patients: a population-based cohort study in the UK. Br J Clin Pharmacol 2018; 84(11): 2551-61

[32] Ceballos-Baumann A. Kontinuierliche Therapie mit der Apomorphinpumpe. Akt Neurol 2011; 38(Supplement 1): S17-S26

[33] Hagell P, Hoglund A, Hellqvist C, et al. Apomorphine formulation may influence subcutaneous complications from continuous subcutaneous apomorphine infusion in Parkinson's disease. J Neurol 2020; 267(11): 3411-7

[34] Sage JI, Trooskin S, Sonsalla PK, et al. Long-term duodenal infusion of levodopa for motor fluctuations in parkinsonism. Ann Neurol 1988; 24(1): 87-9

[35] Olanow CW, Kieburtz K, Odin P, et al. Continuous intrajejunal infusion of levodopa-carbidopa intestinal gel for patients with advanced Parkinson's disease: a randomised, controlled, double-blind, double-dummy study. Lancet Neurol 2014; 13(2): 141-9

[36] Zibetti M, Merola A, Ricchi V, et al. Long-term duodenal levodopa infusion in Parkinson's disease: a 3-year motor and cognitive follow-up study. J Neurol 2013; 260(1): 105-14

[37] Moes HR, Groenendal-Laurensse J, Drent M, et al. Predictors of Time to Discontinuation of Levodopa-Carbidopa Intestinal Gel Infusion: A Retrospective Cohort Study. J Parkinsons Dis 2020; 10(3): 935-44

[38] Buongiorno M, Antonelli F, Camara A, et al. Long-term response to continuous duodenal infusion of levodopa/carbidopa gel in patients with advanced Parkinson disease: The Barcelona registry. Parkinsonism Relat Disord 2015; 21(8): 871-6

[39] Othman M, Widman E, Nygren I, et al. Initial Experience of the Levodopa-Entacapone-Carbidopa Intestinal Gel in Clinical Practice. J Pers Med 2021; 11(4)

[40] Senek M, Nielsen El, Nyholm D. Levodopa-entacapone-carbidopa intestinal gel in Parkinson's disease: A randomized crossover study. Mov Disord 2017; 32(2): 283-6

[41] Senek M, Nyholm D, Nielsen El. Population pharmacokinetics of levodopa gel infusion in Parkinson's disease: effects of entacapone infusion and genetic polymorphism. Sci Rep 2020; 10(1): 18057

[42] Nyholm D, Adnan M, Senek M. Real-Life Use of Levodopa/ Carbidopa Intestinal Gel in Parkinson's Disease According to Analysis of Pump Data. J Parkinsons Dis 2020; 10(4): 1529-34

[43] Uncini A, Eleopra R, Onofrj M. Polyneuropathy associated with duodenal infusion of levodopa in Parkinson's disease: features, pathogenesis and management. J Neurol Neurosurg Psychiatry 2015; 86(5): 490-5

[44] Lehnerer SM, Fietzek UM, Messner M, et al. Subacute peripheral neuropathy under duodopa therapy without cobalamin deficiency and despite supplementation. J Neural Transm 2014; 121(10): 1269-72
[45] Pfister R, Demmel W. Tiefe Hirnstimulation beim idiopathischen Parkinsonsyndrom: Indikationsstellung und Patientenberatung, Nachsorge und postoperative Prognose. Nervenheilkunde 2021; 40: 786-793

[46] Demmel W, Pfister R. Tiefe Hirnstimulation beim idiopathischen Parkinsonsyndrom: Planung, operatives Vorgehen und Risiken. Nervenheilkunde 2021; 40: 794-802

[47] Limousin P, Pollak P, Benazzouz A, et al. Effect of parkinsonian signs and symptoms of bilateral subthalamic nucleus stimulation. Lancet 1995; 345(8942): 91-5

[48] Timmermann L, Jain R, Chen L, et al. Multiple-source current steering in subthalamic nucleus deep brain stimulation for Parkinson's disease (the VANTAGE study): a non-randomised, prospective, multicentre, open-label study. Lancet Neurol 2015; 14(7): 693-701

[49] Nijhuis FAP, Esselink R, de Bie RMA, et al. Translating Evidence to Advanced Parkinson's Disease Patients: A Systematic Review and Meta-Analysis. Mov Disord 2021; 36(6): 1293-307

[50] Dafsari HS, Martinez-Martin P, Rizos A, et al. Eurolnf 2: Subthalamic stimulation, apomorphine, and levodopa infusion in Parkinson's disease. Mov Disord 2019; 34(3): 353-65

[51] Nijhuis FAP, van den Heuvel L, Bloem BR, et al. The Patient's Perspective on Shared Decision-Making in Advanced Parkinson's Disease: A Cross-Sectional Survey Study. Front Neurol 2019; 10: 896

[52] DeLong MR, Huang KT, Gallis ], et al. Effect of advancing age on outcomes of deep brain stimulation for Parkinson disease. JAMA Neurol 2014; 71(10): 1290-5

[53] Sesar A, Fernandez-Pajarin G, Ares B, et al. Continuous subcutaneous apomorphine in advanced Parkinson's disease patients treated with deep brain stimulation. J Neurol 2019; 266(3): 659-66

[54] Slotty P], Wille C, Kinfe TM, et al. Continuous perioperative apomorphine in deep brain stimulation surgery for Parkinson's disease. Br J Neurosurg 2014; 28(3): 378-82

[55] Martins de Campos A, Braz L, Linhares P, et al. Deep brain stimulation for Parkinson's disease: Subcutaneous apomorphine as an alternative for patients unable to tolerate surgery under local anesthesia. J Neurol Sci 2017; 378: 137-9

[56] Foltynie T, Magee C, James C, et al. Impact of Duodopa on Quality of Life in Advanced Parkinson's Disease: A UK Case Series. Parkinsons Dis 2013; 2013: 362908

Bibliografie

Nervenheilkunde 2021; 40: 803-811

DOI 10.1055/a-1592-3610

ISSN 0722-1541

(c) 2021. Thieme. All rights reserved.

Georg Thieme Verlag KG, Rüdigerstraße 14,

70469 Stuttgart, Germany 


\section{Punkte sammeln auf CME.thieme.de}

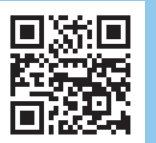

Diese Fortbildungseinheit ist bis zu 12 Monate online für die Teilnahme verfügbar.

Den genauen Einsendeschluss finden Sie beim Modul auf https://cme.thieme.de/CXI76SK.

Sollten Sie Fragen zur Online-Teilnahme haben, finden Sie unter https://cme.thieme.de/hilfe

eine ausführliche Anleitung. Wir wünschen viel Erfolg beim Beantworten

der Fragen!

Unter https://eref.thieme.de/CXI76SK oder über den QR-Code kommen Sie

direkt zum Artikel.

VNR 2760512021160211219

\section{Frage 1}

Welche Antwort ist richtig? Schwankungen in der Levodopawirkung während des Tages...

A betreffen nur die motorischen Symptome.

B gelten als das am meisten störende Symptom bei Patienten mit 6 Jahren und längerer Krankheitsdauer.

C treten typischerweise unmittelbar nach Beginn der ParkinsonKrankheit auf.

D betreffen nicht den Affekt.

E sind eine Kontraindikation für geräteunterstützte Verfahren.

\section{Frage 2}

Was sind Therapieansätze vor der Eskalation zu Medikamentenpumpen und tiefe Hirnstimulation bei Patienten mit Levodopawirkungsschwankungen? Welche Antwort ist falsch?

A Steigerung der Einnahmefrequenz von Levodopa

B Diätetische Maßnahmen: Proteinredistribution, kleine und häufige Mahlzeiten

C Kombinationstherapien von Levodopa mit MAO-B-Hemmer, COMT-Hemmer (Entacapon, Tolcapon, Opicapon) und Dopaminagonisten

D Levodopa und Apomorphin-Pen bedarfsorientiert bei End-ofdose, Delayed-morning-On, Off-Dystonien, und unvorhergesehene Offs

E Quetiapin und Clozapin

\section{Frage 3}

Was trifft auf Apomorphin zu?

A Apomorphin ist vorwiegend ein D2-Agonist.

B Von allen Dopaminagonisten kommt Apomorphin in seiner klinischen Wirkung derjenigen von Levodopa am nächsten.

C Apomorphin wurde jüngst als rektales Antiparkinsonmittel zugelassen.

D Apomorphin wird intravenös über einen Port als Dauerinfusion mittels extern getragener Minipumpe verabreicht.

E Übelkeit, Blutdruckabfall, Müdigkeit werden durch Apomorphin in der Regel besser.

\section{Frage 4}

Was ist die Rationale der jejunalen Infusion eines Levodopa/ Carbidopa intestinalen Gels?

A Die bequeme Darreichungsform von Levodopa.

B Die Umgehung der gestörten Magenentleerung, um Wirkungsfluktuationen zu glätten.

C Die gestörte Schluckfunktion von Parkinson-Patienten.

D Die Verminderung von Halluzinationen.

E Die Möglichkeit auf Carbidopa zu verzichten.

\section{Frage 5}

Welche Aussage zu den Pumpensystemen in der Therapie des fortgeschrittenen Parkinson-Syndroms ist falsch?

A Pumpensystemen unterscheiden sich je nach verwendetem Wirkstoff in Hard- und Software, Größe und Verabreichungsort.

B Neuere Pumpen regeln sich selbst durch Bewegungssensoren.

C In Abhängigkeit von dem Pumpenmodell lassen sich Dosierungen und Extradosen (Bolus) programmieren und Morgenbolus bei Levodopa/Carbidopa intestinales Gel (LCIG) und Levodopa/Entacapon/Carbidopa intestinales Gel (LECIG).

D Es lassen sich kontinuierliche Flussraten und bei manchen Pumpen unterschiedliche Flussraten gebunden an bestimmte Tageszeiten programmieren.

E Es lassen sich Sperrzeiten für Extradosen (Bolus) programmieren.

\section{Frage 6}

Welche Aussage zu der intestinalen Infusion von Levodopa/Carbidopa intestinales Gel (LCIG) und Levodopa/Entacapon/Carbidopa intestinales Gel (LECIG) ist falsch?

A Es braucht eine perkutane endoskopischen Gastrostomie (PEG).

$B$ Es wird eine 2-lumige Sonde verwendet.

C Die äußere Transabdominalsonde endet im Magen. 


\section{Punkte sammeln auf CME.thieme.de}

Fortsetzung $\ldots$

D Die innere feinlumige Applikationssonde sollte durch den Magen und Duodenum nach dem Treitzschen Band in das proximale Jejunum geführt werden.

E Levodopa wird hauptsächlich im Kolon resorbiert.

\section{Frage 7}

Welche Aussage zu den Komplikationen der intestinalen Infusion von Levodopa-Carbidopa ist falsch?

A Dislokation der Applikationssonde nach distal im Jejunum oder proximal zum Magen

B Diskonnektion und Abklemmung der Sonden

C Verstopfung des feinlumigen jejunalen Schenkels in der PEG-Sonde

D Allgemeinen Komplikationen einer PEG wie Wundinfektion bis hin zur Magen-/Darmperforation, Peritonitis und Malnutrition

E Schwerwiegende, teilweise Guillain-Barré-ähnliche Neuropathien sind nicht beschrieben.

\section{Frage 8}

Welche Aussage zur tiefen Hirnstimulation ist falsch?

A Das System für die tiefe Hirnstimulation wird unter die Haut implantiert.

B Die Systeme bestehen aus den stimulierenden Hirnelektroden, Verbindungskabel und dem Impulsgeber mit Batterie.

C Seit einigen Jahren gibt es durch Induktion wieder aufladbare Impulsgeber mit Batterie.

D Eine Veränderung der Stimulationsparameter Pulsbreite, Spannung, Stromstärke, Frequenz der Stimulation erfordert einen operativen Austausch des Impulsgeberaggregats.

E Die Ausbreitung des Stroms kann bei bestimmten Systemen durch eine horizontale Segmentierung der mittleren beiden Ringelektroden in jeweils 3 selektiv steuerbare Kontakte präziser fokussiert werden (direktionale Stimulation).

\section{Frage 9}

Welche Voruntersuchungen bei der Implantation der STN-THS wird nicht gebraucht?

A Eine Kernspintomografie des Gehirns

B Eine FDG-PET-Untersuchung

C Eine neuropsychologische und neuropsychiatrische Untersuchung (Ausschluss Demenz, Abklärung zu Impulskontrollstörungen, u.a.)

D Dokumentation des Ansprechens des Patienten auf Levodopa

E Ausschluss anderer gegen eine STN-Stimulation sprechende Störungen

\section{Frage 10}

Welche Aussage zu der Differenzialindikation und Entscheidung für eine geräteunterstützte Therapie ist falsch?

A Bisher gibt es keine randomisierten kontrollierten Studien, die direkt subkutane Apomorphin-Pumpe, die jejunalen LCIG-/ LECIG-Pumpen und die THS miteinander vergleichen.

B Aspekte wie Verfügbarkeit, Wirtschaftlichkeit, lokale Expertise spielen keine Rolle.

C Präferenzen und Ängste der Patienten und ihrer Bezugspersonen sind wichtig bei der Entscheidung.

D Bei jungen Patienten mit einem aktiven Lebensstil gibt es häufig Vorbehalte gegenüber den Pumpenverfahren aufgrund des Stigmas einer externen Pumpe und des pflegerischen Aufwands.

E Mit einer Besserung von schlecht auf Levodopa ansprechenden Symptomen wie Stimm-, Sprech-, Schluckstörungen und motorischen Blockaden (Freezing) ist nicht zu rechnen. 